\title{
An Image Denoising Algorithm Based On Curvelet Transform
}

\section{Yin kaikai and Su bo}

(College of Electrical Engineering, Henan Polytechnic university, Jiaozuo 454003, China )

\begin{abstract}
Aiming at the limitations of the wavelet transform in image denoising, this paper proposes a new image denoising algorithm based on curvelet transform mathematical method. In this paper, the feasibility of this method is proved by the experimental results. The experiment result shows that, using the proposed new algorithm can get high peak signal to noise ratio, visual effect is very good image.
\end{abstract}

Keywords: denoising, curvelet; algorithm; experimental results.

\section{INTRODUCTION}

In recent years, the theory of wavelet transform has gained great development in the field of image denoising. Although it can analyze one-dimensional signal efficiently, it has limitations in terms of spatial and frequency domain. It can not effectively deal with two-dimensional and above the curve singularity information. For this deficiency, Donoho advanced the curvelet transform theory. Then it has been widely used in the fields of image and signal processing fields. Curvelet transform is actually a multi-level and multi-scale wavelet transform. Firstly, the image is decomposed into different scales; secondly, their line approximation in a straight line after handling with the different scales; thirdly, operating each sub block using ridgelet transform. Analysis of curvelet transform can carry on multi-scale image information, has a significant and far-reaching impact on image processing. Its adaptability is stronger, computing speed is better, compared with the traditional wavelet transform and pure wave threshold de-noising method. This paper presents a new algorithm based on a new generation of the curvelet transform on the theory. Denoising effect of this new algorithm is very good, not only can effectively protect the edge details of image, but also avoid the image blur. In this paper, the new algorithm needs less number of arguments, is simpler to implement.

\section{Basic Theory}

In the Cartesian coordinate system, with $F[T 1, T 2](0=T 1, T<2 n)$ as the input and the discrete form of expression: $C^{D}(j, I, k)=\sum_{0 \leq t_{1}, t_{2} \leq n} f\left[t_{1} t_{2}\right] \phi_{j, l, k}^{D}\left[t_{1} t_{2}\right]$
$\phi\left(w_{1}\right)=\sqrt{\phi\left(w_{1} / 2\right)^{2}-\phi\left(w_{1}^{2}\right)}$ makes $\phi_{j}\left(w_{1}\right)=\phi\left(2^{-j} w_{1}\right)$. The band-pass function is to realize the
multi-scale segmentation. You can get $V_{j}\left(s_{\theta_{1}} w\right)=V\left(2^{[j / 2]} W_{2} / w_{1}-I\right)$ for every $w=\left(w_{1}, w_{2}\right), w_{1}>0 . s_{\theta_{1}}$ is a shear matrix in the formula. $s_{\theta_{1}}=\left[\begin{array}{cc}1 & 0 \\ -\tan \theta_{1} & 1\end{array}\right] \cdot s_{\theta_{1}}$ is not the space, but it must be equidistant slope. When $j=6, I=10$, discrete curvelet transform frequency space of the block diagram as shown below: 


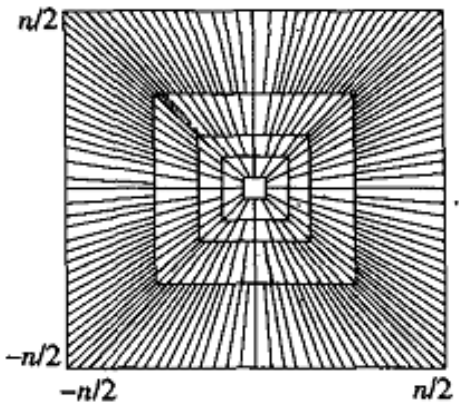

(a)

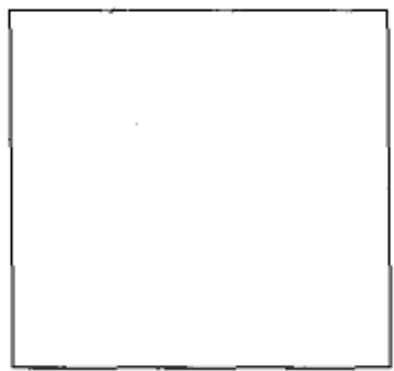

(b)

Figure (a) is a schematic diagram of the discrete curvelet frequency domain. Figure (b) is the segmentation scale of $\mathrm{j}$ =6wave frequency domain. Definition: $\tilde{U}_{j}(w)=\phi_{j}\left(w_{1}\right) V_{j}(w)$, we can get $\tilde{U}_{j, l}(w)=\phi_{j}\left(w_{1}\right)$ and $V_{j}\left(s_{\theta_{1}} w\right)=\tilde{U}_{j}\left(s_{\theta_{1}} w\right)$ for every $\theta_{1} \in[-\pi / 4, \pi / 4]$. At the same time, meet the following formula: $\sum_{I=-\infty}^{\infty}\left|V_{j}\left(s_{\theta_{1}} w\right)\right|^{2}=1, \sum_{\text {al I scal e al l angl es }}\left|\tilde{U}_{j, I}(w)\right|^{2}=1$

\section{Curvelet Transform Denoising Method}

We briefly introduce the basic algorithm for image denoising based on discrete curvelet transform. Its core idea is that for each region to use periodic technology will be its one one mapped to the affine region on the origin. Set the original image of $F(m, n)$. The specific algorithm is as follows: The first step: on two-dimensional function of fast Fourier transform, the frequency domain expression for $\mathrm{F}$ goes [n1, N2, N / $2<\mathrm{N} 1, \mathrm{~N} 2$ is less than or equal to N / 2 in Cartesian coordinates.

The second step: in the frequency domain, for each pair $(\mathrm{J}, \mathrm{I})$, were re sampling[n1 n2],can get the sample value: $\hat{f}\left[n_{1}, n_{2}-\right.$ $\left.n_{1} \tan \theta_{l}\right],\left(n_{1}, n_{2}\right) P_{j}$

The third step: after the interpolation function $\mathrm{f}^{\wedge}$ and window function multiplication, can be obtained: $\hat{f}\left[n_{1}, n_{2}\right]=\hat{f}\left[n_{1}, n_{2}\right.$ $\left.-n_{1} \tan \theta_{l}\right] U_{j}\left[n_{1}, n_{2}\right]$ 。

The fourth step: localized $\mathrm{f}^{\wedge}$ ( $\mathrm{n} 1$, and $\left.\mathrm{n} 2\right)$ around point wrapping.

The fifth step: getting the discrete curvelet coefficient set $c^{D}(j, l, k)$ through the 2DIFFT transform.

The sixth step: to curvelet coefficient set inverse transformation, we can get estimates of the image. At this point, we get the denoised image.

This algorithm can effectively improve the numerical image quality and objective evaluation index PSNR, but the following two issues remain unresolved:

The first: Most of the image information are reflected in the image edge information, in the use of the curvelet transform, we usually choose the appropriate threshold to reduce image noise. Because the curvelet transform is not has translation invariance, so no matter adopt what kind of threshold, will be on the coefficient of excess strangled, eventually lead to the edge of the image appears "ringing" effect. This is the pseudo Gibbs phenomenon.

The second: curvelet transform has certain correlation between linear characteristic makes the transform coefficient, as long as the coefficient of change, will cause the airspace corresponds to a line in all the values of the corresponding change, the image can produce linear distortion. This is called the "scratch" distortion. 


\section{ISSN $2347-1921$ \\ Volume $12 \mathrm{Number} 1$ \\ Journal of Advances in Mathematics}

\section{A new image denoising algorithm is proposed in this paper}

For the two problems existing in the above algorithm, this paper targeted improvement. First: with the noise of image threshold de-noising for translation invariant first, combining cycle translation method to eliminate pseudo "gibbs" phenomena of the image. The second: using iterative computing method, approximate target image, thereby eliminating scratches the distortion image space domain. Specific steps of the algorithm are as follows:

First step: the noise image first to convert the inverse fast Fourier transform, fast Fourier transform, for translation is cycle.

The second step: we can get the curvelet coefficient of different scales by curvelet transform, and then monte carlo method is used to estimate the noise variance. The threshold proportion coefficient signal at the coarsest scale at 2.2 , in the fine scale at 2.5. This scale with curvelet coefficients, get the inverse curvelet transform coefficients.

The third step: to get the coefficient of curvelet transform, we can get the curvelet coefficient of temporary refactoring. The inverse curvelet transform coefficient is obtained.

The fourth step: steps for the second and third in the correlation coefficient for iterative arithmetic and reverse cycle again translation operations, the reconstruction of the synthetic images, eventually have to noise reduction of image information.

\section{5 experiments and results analysis}

The image joined the mean is zero gaussian white noise, the noise variance is 20 , compared the wavelet image noise reduction, curvelet transform de-noising algorithm and the image noise reduction algorithm is put forward in this paper, finally, the processed image data analysis. Processing the results as shown in the figure below:

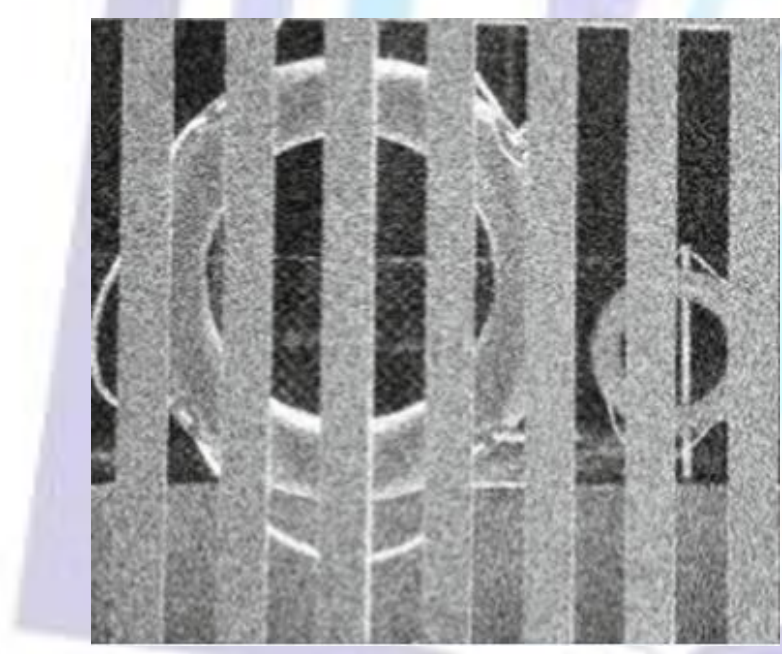

(a) Gray image after adding noise

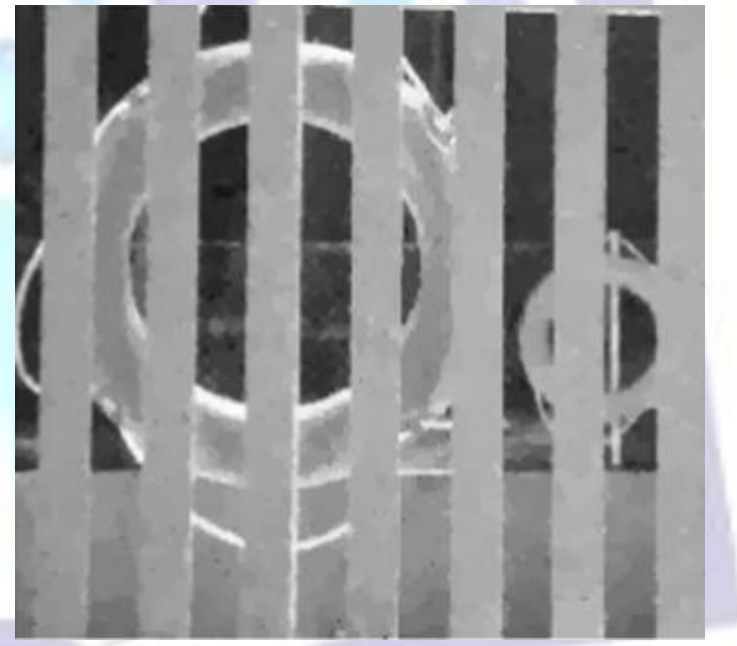

(b) Image denoising by wavelet transform 


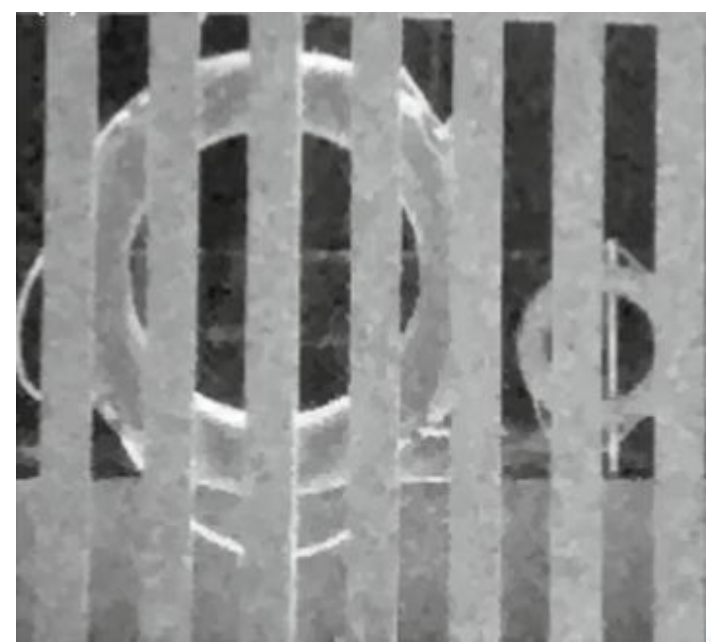

(c) Image denoising by curvelet transform

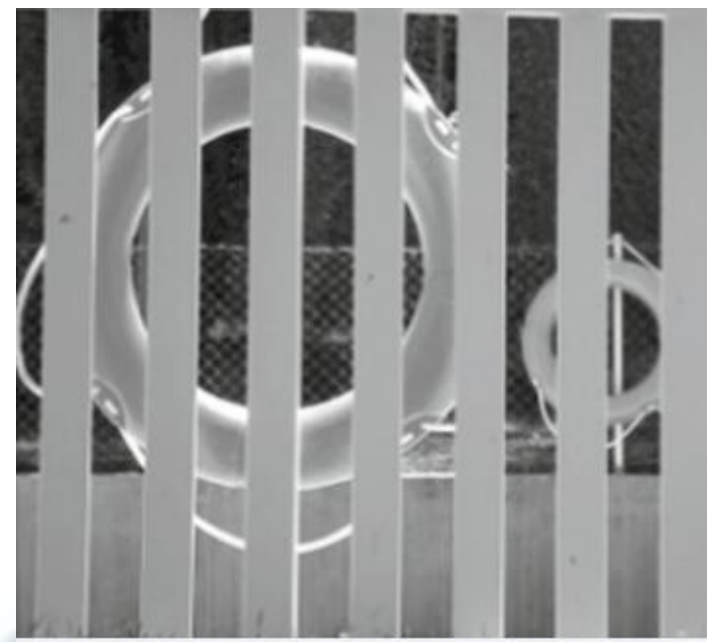

(d) Image denoising new denoising algorithm

The peak signal-to-noise ratio (PSNR) is internationally recognized as a standard formula as follows: $p s n r=10 \lg \left[\frac{255^{2}}{\frac{1}{M \times N} \sum_{m=0}^{M-1} \sum_{\mathrm{n}=0}^{\mathrm{N}-1}\left(\mathrm{x}(\mathrm{m}, \mathrm{n})-\mathrm{x}_{\mathrm{d}}(\mathrm{m}, \mathrm{n})^{2}\right)}\right]$.

The larger value shows better denoising effect of image, The results are shown in Table 1.

\begin{tabular}{|c|rrrrr|}
\hline $\begin{array}{c}\text { Comparison of the } \\
\text { experimental data }\end{array}$ & Original & Noise wavelet & curvelet & new algorithm \\
\hline PSNR & 32.2670 & 20.8173 & 32.0952 & 32.1065 & 33.2131 \\
\hline
\end{tabular}

Table 1:Several common methods of noise reduction on the recovery of the image PSNR value comparison.

According to the data in Table 1, new algorithm processing including noise of image, the recovery effect is very good. The image edge information is greatly improved and retention, but also reduces the "scratch" distortion. Greatly improve the peak signal-to-noise ratio index. It has best denoising effect among the three. But it is the longest processing time, it is about 5 times that of the curvelet algorithm.

\section{Summary and Outlook}

New algorithm strengthen the image denoising effect, better reduces the curvelet noise reduction leads to the "scratch" image distortion, also preserve the image edge information. It can also show good performance in different strength under the influence of noise.

\section{Reference}

[1] Z. M. Li, Y. W. Lin, J. Huang. Preprocess Algorithm of PCB Line Detection[J].Optics andPrecision Engineerin, 2007, 15(2):242-246

[2] M. Moganti, F. Ercal. Automaticprinted Circuited Board Inspection Systems[J].IEEE Potentials,1995, 14(3): $6-10$

[3] H. G. da Silva, T. G. Amaral, O. P. Dias. Automatic Optical Inspection for Detecting Defective

Solders on Printed Circuit Boards[C].Annual Conference on IEEE Industrial Electronics Society, 
Melbourne, 2010, 36: 1087-1091

[4] N. Talbot. The Use of Automated Optical Testing (AOT) in Statistical Process Control (SPC) forPrinted Circuit Board (PCB) Production [J].Circuit World, 2003, 29(4): 19 22

[5] R. F. Sajal, M. Kamruzzamman, F. Ahmed Jewel.Machine Vision Based Automatic System for Real Time Recognition and Sorting of Bangladeshi Bank Notes[C]. International Conference on Computer and Information Technology, 2008, 356-358

[6] T. T. Chung, Y. C. Huang. Optimum Desisn of A high Precision AOI Machine Structure[C]. International Conference on Mechanical and Electronics Engineering, 2010, 164-168

[7] N. Mar, P. Yarlagadda, C. Fookesc. Design and Development of Automatic Visual Inspection

System for PCB Manufacturing [J]. Robotics and Computer Integrated Manufacturing, 2011,27(5):949-962

[8] S. Kishimoto, N. Kakimori, Y. Yamamoto, et al.. A printed Circuit Board (PCB) Inspection

System Employing the Multi-lighting Optical System[C]. 8th IEMT International Electronic

Manufacturing Technology Conference, IEEE, 1990, 120-129

[9] W. Y. Wu, M. J. J. Wang, C. M. Liu, et al. Automated Inspection of Printed Circuit Boards through Machine Vision [J]. Computer in Industry, 1996, 28(2):103-111

[10] H. Zheng, L. X. Kong, S. Nahavandi. Automatic Inspection of Metallic Surface Defects Using Genetic Algorithms [J]. Journal of Materials Processing Technology, 2002, 12(6):427-433

[11] D. Z. WANG, C. H. WU, I. ANDREW, et al.. Fast Multi-Template Matching Using a Particle

Swarm Optimization Algorithm for PCB Inspection[C]. Conference European Workshops on the Theory and Applications of Evolutionary Computation, 2008, 365-370

[12] E. K. Teoh, D. P. Mital, B. W. Lee, et al.. Automated Visual Inspection of Surface Mount PCB

[J]. Signal Processing and System Control Factory Automation, 1990, 20(8):576-580

[13] HAE-WON OH, TAE-HYUNG PARK. Gerber-character Recognition System of Auto-teaching Program for PCB Assembly Machines[C]. SICE Annual Conference, 2004, 8(2):300-30

[14] Zhang, X.P., Desai, M.D.: Adaptive denoising based on SURE risk. In: IEEE Signal Process. Lett. 5(10), 265-267 (1998)

[15] Chang, S.G., Yu, B., Vetterli, M.: Adaptive wavelet thresholding forimage denoising and compression. In: IEEE Trans. Image Process.9(9), 1532-1546 (2000)

[16] Cai, T.T., Silverman, B.W.: Incorporating information on neighboring coefficients into wavelet estimation. Sankhya, Ser. B 63(2),127-148 (2001)

[17] Cai, T.T., Zhou, H.H.: A data-driven block thresholding approachto wavelet estimation. Ann. Statist. 37(2), 569-595 (2009)

[18] Sendur, L., Selesnick, I.W.: Bivariate shrinkage functions forwavelet-based denoising exploiting interscale dependency. In: IEEE Trans. Signal Process. 50(11), 2744-2756 (2002) 